DOI: https://doi.org/10.11144/Javeriana.upsy17-3.rcdt

\title{
Renovación contextual de la distribución temporal del responder ${ }^{*}$ Contextual Renewal of Temporal Patterns of Responding
}

\author{
José Natividad Moreno Zazueta \\ Universidad de Guadalajara, México \\ ORCID: http://orcid.org/0000-0001-9532-7695 \\ Carlos Javier Flores Aguirre ${ }^{\mathrm{a}}$ \\ Universidad de Guadalajara, México \\ ORCID: http://orcid.org/0000-0002-3085-4029
}

a Autor de correspondencia. Correo electrónico:
carlos.faguirre@academicos.udg.mx

Para citar este artículo: Moreno, J. N. \& Flores, C. J. (2018). Renovación contextual de la distribución temporal del responder. Universitas Psychologica, 17(3), 1-10. https://doi.org/10.11144/Javeriana.upsy 17-3.rcdt

\section{RESUMEN}

Se ha reportado que en ciertos fenómenos (recuperación espontánea, readquisición, resurgencia y renovación contextual) es posible observar la recuperación de una respuesta previamente extinguida. La renovación contextual, se refiere a la reaparición de una respuesta extinguida que resulta de un cambio de contexto entre la fase de extinción y prueba. El propósito del presente trabajo consistió en evaluar la renovación contextual, atendiendo tanto a la distribución temporal del responder, como a la tasa de respuesta. Se encontró una recuperación de la tasa de respuesta, siendo un resultado consistente con los reportados en el área de renovación contextual. Además, se pudo identificar una renovación de la distribución temporal de respuesta. Los resultados se discuten reconociendo que la tasa y la distribución temporal constituyen dos dimensiones de la ejecución que son moduladas por los cambios de contexto.

Palabras clave

Adquisición; extinción; renovación contextual; intervalo fijo; ratas.

\begin{abstract}
It has been reported that in certain phenomena (spontaneous recovery, reacquisition, resurgence and contextual renewal) it is possible to observe the recovery of a previously extinguished response. Contextual renewal refers to the reappearance of an extinguished response that result from a change of context between the extinction and testing phase. The purpose of the present study was to evaluate contextual renewal, considering both the temporal distribution of the responses and the response rate. A recovery of the response rate was found, being a result consistent with those reported in the area of contextual renewal. In addition, a renewal of the temporal distribution of the responses could be identified. The results are discussed by recognizing that rate and temporal distribution are two behavioral dimensions that are modulated by contextual changes.

Keywords

Acquisition; extinction; contextual renewal; fixed interval; rats.
\end{abstract}


La renovación contextual, se refiere a la reaparición de una respuesta extinta que resulta de un cambio de contexto entre la fase de extinción y prueba (Bouton \& Bolles, 1979; Podlesnik, Kelley, Jimenez-Gomez, \& Bouton, 2017). En la literatura se pueden identificar tres diseños que permiten dar cuenta de este fenómeno: 1) cuando se establecen un conjunto de señales (olfativas, táctiles, visuales, etc.) durante la fase de adquisición (A), posteriormente los sujetos se exponen a un conjunto de nuevas señales durante la fase de extinción (B) y finalmente, durante la fase de prueba se regresa a los sujetos al conjunto de señales que estaban presentes durante la fase de adquisición, a este diseño se le conoce como diseño ABA (Eddy, Todd, Bouton \& Green, 2016; Kearns \& Weiss, 2007; Podlesnik \& Miranda-Dukoski, 2015; Sweeney \& Shahan, 2015; Todd, Winterbauer \& Bouton, 2012; Trask \& Bouton, 2016; Vurbie, Gold \& Bouton, 2011); 2) cuando el conjunto de señales de adquisición y extinción son las mismas (A) y solo cambian durante la fase de prueba (B), se le conoce como diseño AAB (Bernal-Gamboa, Carrasco-López \& Nieto, 2014; Bouton, Todd, Vurbic, \& Winterbauer, 2011; Nakajima, Tanaka, Urushihara, \& Imada, 2000); 3) cuando el conjunto de señales es diferente para adquisición (A), extinción (B) y prueba (C), se le conoce como diseño ABC (Cuevas, Learmonth \& Roveer-Collier, 2015; Todd et al., 2012).

Utilizando un diseño ABA y una preparación de condicionamiento operante, Kelley, Linddon, Ribeiro, Greif y Podlesnisk. (2015) demostraron la renovación contextual tanto en palomas como en niños. En el Experimento 1, las palomas fueron expuestas a un programa de intervalo fijo (IF), mientras que en el Experimento 2 los niños fueron entrenados con un programa de reforzamiento continuo. En ambos experimentos los autores observaron un incremento en la tasa de respuesta posterior a la extinción.

En estudios recientes, se ha reportado la renovación contextual utilizando preparaciones de condicionamiento operante y utilizando la tasa de respuesta como medida (Cuevas et al.,
2015; Eddy et al., 2016; Podlesnik \& MirandaDukoski, 2015; Sweeney \& Shahan, 2015; Trask $\&$ Bouton, 2016), estos estudios muestran la generalidad del fenómeno a través de distintos programas de reforzamientos y distintas especies, siendo ABA el diseño más utilizado.

Sidman (1960) menciona que una de las cuestiones que se debe tomar en cuenta sobre los hallazgos experimentales, es la generalidad. En este sentido, la generalidad se puede observar de varias maneras, por ejemplo: en un sujeto experimental (resultado representativo para otros organismos de la misma especie), entre especies (resultado representativo en otros organismos de diferentes especies) y de las variables (resultado representativo para un conjunto de condiciones cada vez más amplio). La generalidad es el punto de referencia a partir del cual los científicos, pueden evaluar el grado de verificación aportado por cualquier extensión dada de las condiciones originales.

En la renovación contextual, la generalidad de las variables, en especial la variable dependiente, se ha centrado en la tasa respuesta, pero existen otras propiedades de la respuesta que se pueden reportar como variable dependiente (Gilbert, 1958). Por ejemplo, la distribución del responder (localización temporal o espacial donde se emiten las respuestas). Para algunos investigadores, la distribución temporal del responder es considerada un factor definitorio de la ejecución, al igual que la tasa de respuesta (Guilhardi \& Church, 2006; Skinner, 1938; Ferster \& Skinner, 1957). Además, para algunos investigadores (Gentry, Weiss \& Laties, 1983) los resultados basados simplemente en el conteo de respuestas, pueden tener un valor limitado debido a que la forma o distribución temporal del responder, no es considerada cuando únicamente se reporta la tasa de respuesta.

Una distribución del responder sobre la que se han generado diversas investigaciones, es la producida por el programa de reforzamiento IF (Cabrer, 1987; Cançado \& Lattal, 2011; Dews, 1969; Ferster \& Skinner, 1957; Guilhardi \& Church, 2004; López \& Menez, 2005), la cual se caracteriza por una pausa al inicio del intervalo y un incremento acelerado del responder conforme 
se aproxima el final del intervalo. El uso de este programa de reforzamiento, se ha empleado en preparaciones experimentales para el estudio de la renovación contextual (Kelley et al. 2015). Sin embargo, en el estudio de Kelley et al. (2015), únicamente se reportó la tasa de respuesta. Por otro lado, en estudios sobre recuperación espontánea (Delamater, 2016), readquisición (Guilhardi \& Church, 2006) y resurgencia (Cançado \& Lattal, 2011) han utilizado este programa de reforzamiento y se ha podido identificar la recuperación de la ejecución (tasa y distribución temporal del responder), mostrando cierto grado de generalidad. Por lo anterior, es posible pensar que en un estudio de renovación contextual, también se podría identificar la recuperación de una distribución temporal del responder. El presente estudio, se diseñó con el propósito de identificar la renovación contextual tanto de la tasa de respuesta como de la distribución temporal del responder, utilizando un diseño ABA. Un estudio de esta naturaleza, es importante porque permitiría reconocer que también es posible que la distribución temporal del responder se recupere y no únicamente la frecuencia del responder, contribuyendo al reconocimiento de que ambas propiedades del comportamiento (frecuencia y distribución temporal) son dos dimensiones susceptibles de renovación contextual.

\section{Método}

Sujetos

Se utilizaron ocho ratas Wistar macho de aproximadamente tres meses de edad (con un rango de $280 \mathrm{~g}-330 \mathrm{~g}$ ) al inicio del experimento e ingenuas experimentalmente, privadas de agua por un periodo de 23 horas y con acceso libre durante 30 minutos después de cada sesión experimental. Las ratas estuvieron alojadas en jaulas individuales (largo: $37 \mathrm{~cm}$, ancho: $27 \mathrm{~cm}$, alto: $17 \mathrm{~cm})$, con comida disponible, en un ciclo de luz/oscuridad de 12/12 horas.

\section{Aparatos}

Se usaron ocho cámaras experimentales marca MED Associates (ENV-008) para ratas. Cada cámara tenía en el panel operativo una apertura rectangular situada en el centro a $2 \mathrm{~cm}$ por encima del suelo, un dispensador de agua (ENV-202M) detrás de la apertura, una palanca retráctil al lado derecho de la apertura a 2.5 $\mathrm{cm}$ del piso, una lámpara (3 W) que estaba situada en el centro superior de la pared trasera proporcionando iluminación general a la cámara. Se necesitaba de una fuerza de $0.25 \mathrm{~N}$ en la palanca para cerrar el microswitch y que hiciera entrega del reforzador. Como reforzador, se utilizó una gota de agua de 0.02 cc que se presentó por medio de la activación del dispensador durante 3s. Cada cámara experimental, contaba con distintas señales contextuales (visuales, olfativas y táctiles) y se colocó dentro de un cubículo de aislamiento acústico (ENV-022M) con un ventilador que funcionó como ruido blanco y facilitaba la circulación del aire al interior de la cámara. La programación, el registro y la colección de eventos se realizó mediante un equipo de cómputo, una interfaz y el software MED-PC IV para Windows.

\section{Señales contextuales}

Se conformaron dos conjuntos de cámaras, de cuatro cámaras cada uno, las cuales se caracterizaron por las señales contextuales correspondientes a cada conjunto. Para un conjunto (A) cuatro cámaras experimentales tenían por fuera un recipiente con una bolita de algodón, impregnada de líquido con olor a pinol. En el suelo, se colocó una hoja de acrílico que cubrió toda la superficie, en las paredes y techo se colocaron líneas negras en diagonal de dos centímetros sobre un fondo blanco con un espacio de dos centímetros entre líneas. El otro conjunto de cuatro cámaras experimentales (B), tenían un recipiente con una bolita de algodón impregnada de líquido olor manzana-canela, en el suelo se colocó malla de gallinero que cubrió toda la superficie, en las paredes y techo se 
colocó una hoja con círculos negros de cinco centímetros de diámetro sobre un fondo blanco.

\section{Procedimiento}

Para todos los sujetos, se estableció la respuesta de presión a la palanca utilizando un programa de reforzamiento continuo y un programa de Tiempo Fijo (TF) $30 \mathrm{~s}$ que operaban de manera simultánea; es decir, cada presión a la palanca producía una gota de agua y de manera concurrente se podía activar el bebedero cada 30 $s$ independientemente de la respuesta. Una vez que obtenían 100 entregas de agua, los sujetos pasaron a un programa de IF 7 s y que en sesiones consecutivas se fue incrementando hasta 27 s (7 s, 14 s, 21 s y 27 s). Los sujetos se expusieron durante dos sesiones a cada valor del IF. Al concluir el entrenamiento, las ratas fueron asignadas aleatoriamente a uno de los dos grupos; cuatro para el Grupo ABA y cuatro para el Grupo $\mathrm{ABB}$.

\section{Adquisición}

Todos los sujetos, fueron expuestos a un programa de IF30 s en el conjunto de cámaras experimentales 'A'. Al inicio de la sesión se encendía la luz general y se extendía la palanca. Las sesiones tuvieron una duración de 30 minutos, los sujetos permanecieron en esta condición por un mínimo de 10 sesiones y hasta cumplir con un criterio de estabilidad, que consistió en que la tasa de respuestas no variara \pm el $10 \%$ del promedio de las tres sesiones anteriores.

\section{Extinción}

En esta fase, todos los sujetos fueron expuestos al conjunto de cámaras experimentales 'B' y se mantuvieron en las mismas condiciones a las de la fase anterior, excepto que se eliminó la entrega del agua. Los sujetos, concluyeron esta fase una vez que la tasa de respuesta fue menor al 10\% de la tasa de respuesta obtenida en la última sesión de adquisición. Las sesiones tuvieron una duración de 30 minutos.

Prueba

Para los sujetos del Grupo ABA, la prueba se realizó en el conjunto de cámaras experimentales 'A'; mientras que para los sujetos del Grupo $\mathrm{ABB}$, se realizó en el conjunto de cámaras experimentales 'B'. Para el Grupo ABA, las condiciones fueron las mismas que durante la fase de adquisición, con la diferencia que no se entregaba el reforzador. El Grupo ABB, tuvo las mismas condiciones que en la fase anterior. Todos los sujetos tuvieron una sesión de prueba con una duración de 30 minutos.

\section{Resultados}

En la Tabla 1 se presenta el número de sesiones que requirió cada uno de los sujetos para cumplir el criterio de estabilidad durante las fases 1 y 2 . Los sujetos del Grupo ABA requirieron entre 17 y 21 sesiones durante la adquisición, mientras que los sujetos del Grupo ABB requirieron entre 14 y 22 sesiones. Durante la extinción los sujetos del Grupo ABA requirieron entre 2 y 3 sesiones, mientras que los del Grupo ABB entre 2 y 4.

\section{Tabla 1}

Numero de sesiones requeridas por sujeto para cumplir el criterio de estabilidad durante la Fase de Adquisición y Extinción

\begin{tabular}{|ccrr}
\hline \multirow{2}{*}{ Grupo } & \multirow{2}{*}{ Sujetos } & \multicolumn{2}{c}{ Sesiones } \\
\cline { 3 - 4 } & & Adquisición & Extinción \\
\hline \multirow{4}{*}{ ABA } & SU1 & 21 & 2 \\
& SU2 & 14 & 3 \\
& SU3 & 20 & 2 \\
& SU4 & 17 & 2 \\
\hline \multirow{4}{*}{ ABB } & SU5 & 14 & 2 \\
& SU6 & 22 & 2 \\
& SU7 & 14 & 4 \\
& SU8 & 15 & 2 \\
\hline
\end{tabular}

Una de las variables dependientes más importantes en los estudios de renovación contextual, es la tasa de respuesta. Es por ello, 
que en la Figura 1 se presenta la tasa de respuesta por minuto (mediana) de la última sesión de cada grupo durante la fase de adquisición, la extinción y la prueba. Durante la adquisición, la tasa de respuesta para el Grupo ABA fue de 9.71 (DS $=3.63)$, mientras que para el Grupo ABB fue de 10.76 (DS = 4.88); una prueba $U$ de Mann-Whitney para muestras independientes confirmó que no se encontraron diferencias significativas entre grupos, $U=7, p>0.05$. Durante la extinción, se observó un decremento en la tasa de respuesta para ambos grupos; para el Grupo ABA fue de 0.28 (DS = 0.08), mientras que para el Grupo $\mathrm{ABB}$ fue de 0.15 $(D S=0.17)$, tampoco se encontraron diferencias estadísticamente significativas, $U=5, p>0.05$. En la prueba se observó que la tasa de respuesta para el Grupo ABA fue 1.70 (DS = 1.49), mientras que para el Grupo ABB fue 0.50 (DS = 0.13), la prueba estadística confirmó diferencias entre grupos, $U=0, p<0.05$.

\section{Figura 1}

Tasa de respuesta (mediana) y desviación estándar de la última sesión de cada grupo durante cada una de las fases experimentales. Grupo ABA (barras en color negro), Grupo $A B B$ (barras en color gris)

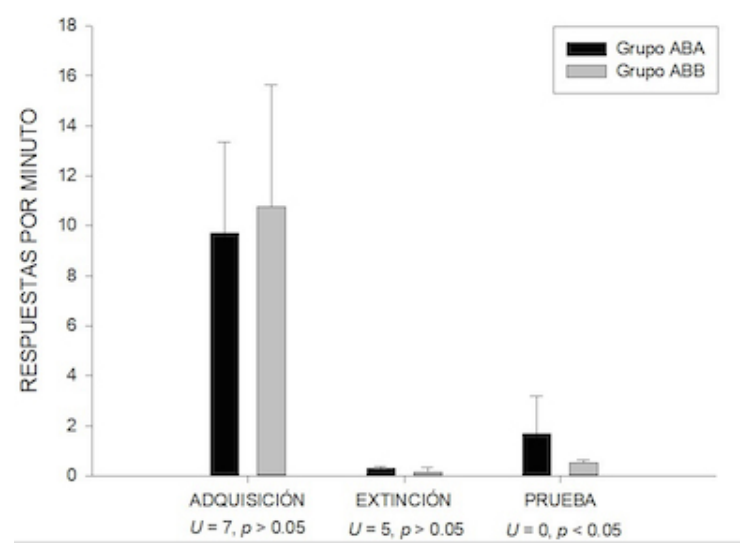

En la Figura 2 se presenta la distribución temporal de las respuestas en subintervalos de 3 s durante la última sesión de adquisición, extinción y prueba de los sujetos del Grupo ABA; las distribuciones se muestran en una escala de proporción de la tasa máxima. Es decir, el valor de cada subintervalo se dividió en el subintervalo con mayor valor (e.g., una rata emite cuatro respuestas en el subintervalo 5 , siete respuestas en el subintervalo 7 y veinticuatro respuestas en el subintervalo 10, en este caso todos los subintervalos se dividirán entre 24 , debido que para este sujeto veinticuatro respuestas fue la tasa máxima). De manera general, se puede observar que en la adquisición los sujetos tienden a incrementar su responder, conforme transcurre el intervalo mostrando una distribución tipo festón. Durante la extinción, se perciben respuestas aisladas al interior del intervalo y que la distribución tipo festón desaparece. Es así como durante la prueba se observa, de manera general que la distribución temporal del responder es similar a la observada durante la adquisición, reapareciendo el patrón de ejecución tipo festón.

\section{Figura 2}

Distribución de las respuestas en subintervalos de 3 segundos (proporción de la tasa máxima), cada fila representa un sujeto del Grupo ABA. Las columnas muestran la última sesión de cada fase; adquisición (izquierda), extinción (centro), y prueba (derecho). En el interior de cada gráfica también se muestra en la parte superior izquierda de cada grafica la tasa de respuesta de la última sesión de cada fase

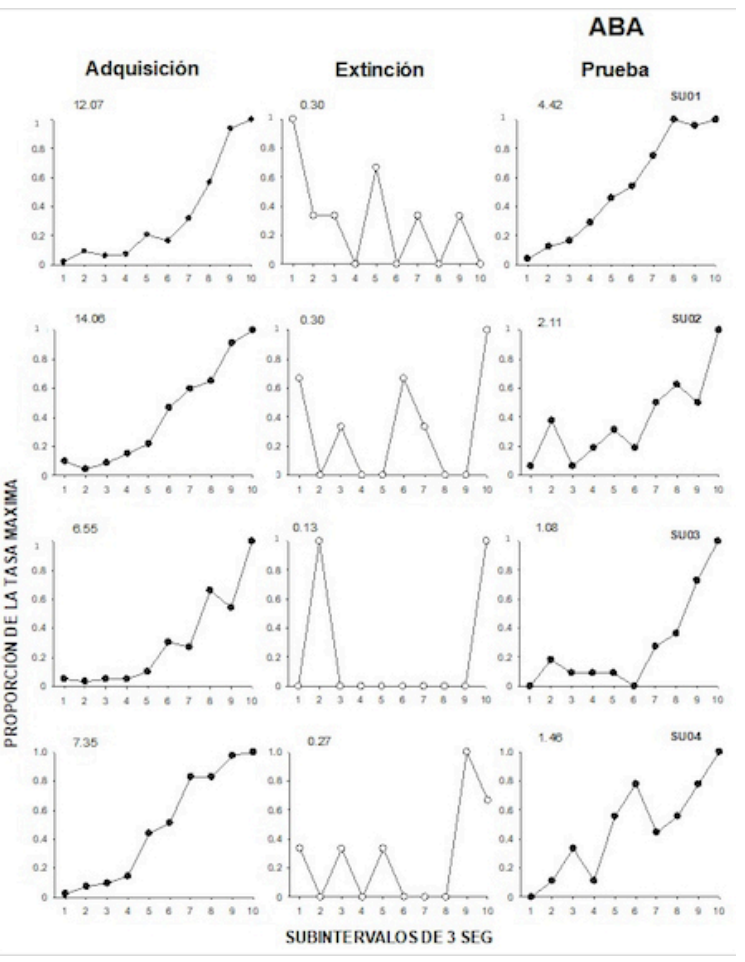


En la Figura 3 se presenta la distribución temporal de las respuestas en subintervalos de 3 s (proporción de la tasa máxima), en la última sesión de adquisición, extinción y prueba de los sujetos del Grupo ABB. De la misma manera que para los sujetos del Grupo ABA, en los sujetos del Grupo ABB se observó que durante la adquisición, los sujetos tienden a incrementar su responder conforme transcurre el intervalo. Durante la extinción, se observan respuestas aisladas al interior del intervalo y que la distribución tipo festón, desaparece al igual que los sujetos del Grupo ABA. A diferencia de lo observado en los sujetos del Grupo ABA, durante la prueba se puede observar que la distribución temporal del responder no fue similar a la observada durante la adquisición.

Para determinar de manera cuantitativa la diferencia o similitud entre las distribuciones temporales del responder obtenidas durante la fase de adquisición y de prueba de cada sujeto, se realizaron correlaciones mediante la $r$ de Pearson. Se encontró que la correlación fue de 0.85 para el Grupo ABA y de - 0.04 para el Grupo ABB. Con base en los valores de la $\mathrm{r}$ de Pearson se realizó una prueba $U$ de Mann-Whitney para muestras independientes, la cual confirmó diferencias estadísticamente significativas entre grupos, $U=0, p<0.05$.
Figura 3

Distribución de las respuestas en subintervalos de 3 segundos (proporción de la tasa máxima), cada fila representa un sujeto del Grupo ABB. Las columnas muestran la última sesión de cada fase; adquisición (izquierda), extinción (centro), y prueba (derecho). En el interior de cada gráfica también se muestra en la parte superior izquierda de cada grafica la tasa de respuesta de la última sesión de cada fase

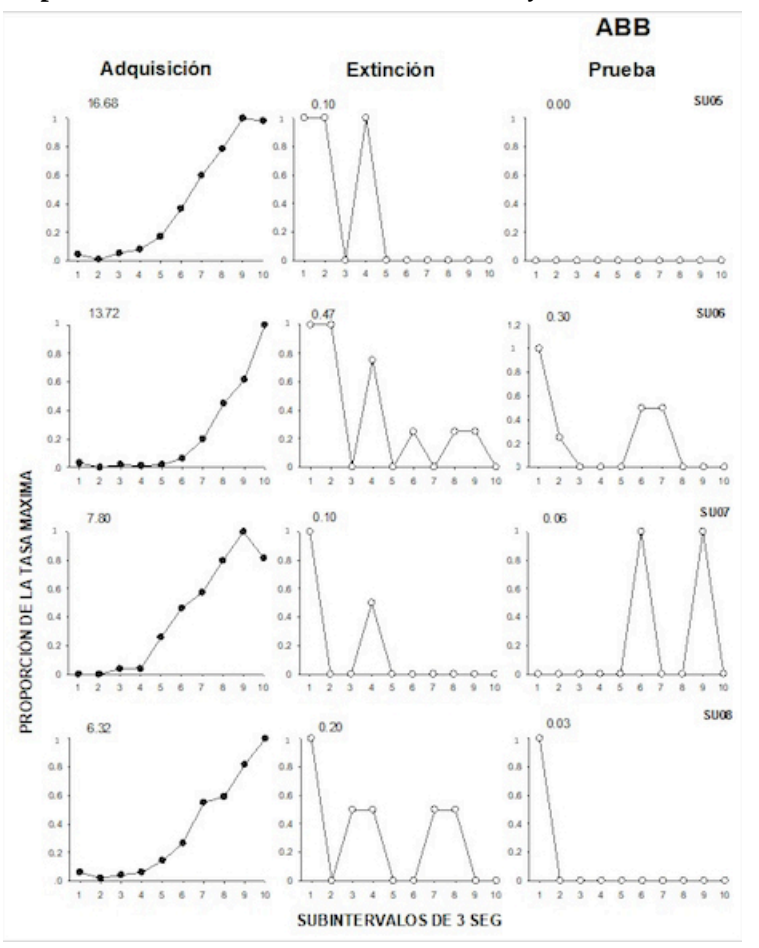

\section{Discusión}

En el presente estudio se examinó la renovación contextual tanto de la tasa de respuesta como de la distribución temporal del responder. En la fase de adquisición, se observó variabilidad de la tasa de respuesta entre los sujetos, sin embargo, todos mostraron un incremento en su responder conforme avanzaba el intervalo, los sujetos mostraron una distribución típica del IF (festón). Estos resultados son consistentes con estudios previos (Cabrer, 1987; Dews, 1969; Ferster \& Skinner, 1957; Guilhardi \& Church, 2004; López \& Menez, 2005), en los que se notaron este mismo tipo de ejecución al emplear programas de IF. En la fase de extinción la tasa 
de respuesta, disminuyó en comparación con la observada durante la fase de adquisición, no obstante, se examinaron respuestas aisladas al interior del intervalo. Para la prueba se analizó una recuperación de la tasa de respuesta, este resultado es consistente con los reportados en el área de renovación contextual con un diseño ABA (Bouton et al. 2011; Nakajima et al. 2000). Además, fue posible reconocer la recuperación de la distribución temporal del responder típico del programa IF, extendiendo los hallazgos en el área de renovación contextual ABA.

Estos resultados son consistentes con otros trabajos en los que también se observó una recuperación en la distribución temporal del responder después de la extinción en preparaciones para estudiar la recuperación espontánea, readquisición y resurgencia (Cançado \& Lattal, 2011; Delamater, 2016; Guilhardi \& Church, 2006).

Por ejemplo, en un estudio de resurgencia, Cançado y Lattal (2011) utilizaron palomas, estas cuales fueron expuestas a un programa múltiple IF-IV. Los componentes del programa fueron señalados por una luz de color (blanco o verde) en la tecla central; en donde los sujetos picaban para obtener alimento, según el programa vigente. Una vez que los sujetos mostraban el patrón de respuesta correspondiente a cada programa, pasaban a la siguiente fase. Durante la fase de extinción, las luces del programa múltiple IF-IV seguían en la tecla central, a excepción de las respuestas que dejaron de ser reforzadas. En esta etapa, se implementó un programa IV en la tecla de la derecha, la cual se iluminaba con una luz de color azul. Esta condición se mantuvo hasta que los sujetos presentaron una tasa de respuesta menor a una respuesta por minuto en la tecla central. Una vez que se cumplía el criterio establecido, los sujetos pasaron a la fase de prueba en la que se mantuo las mismas condiciones que la fase anterior, con la excepción de que tanto las respuestas en la tecla central (programa múltiple IF-IV) como aquellas respuestas en la tecla de la derecha (IV) no eran reforzadas. El reporte arrojó que los sujetos tuvieron un incremento de la tasa de respuesta obtenida en la tecla central, durante la prueba, en comparación con la tasa de respuesta de extinción. Además reportaron la recuperación de la distribución temporal del responder, correspondiente a cada componente del programa múltiple de la tecla central (IF-IV).

Por otro lado, en un estudio de readquisición Guilhardi y Church (2006) utilizaron ratas, las cuales estuvieron en tres programas de reforzamiento de Intervalo Fijo (IF): 30 s, 60 s y 90 s, cada uno asociado a una señal que fungía como estímulo discriminativo (ruido blanco, luz o clicker). Posteriormente, durante la fase de extinción, el procedimiento fue similar al de la fase anterior, con la diferencia de que no se entregaba el alimento. Por último, en la fase de prueba se crearon dos grupos: uno llamado consistente y otro denominado inconsistente, para todos los sujetos se reforzaron las respuestas dependiendo del IF vigente. Para el grupo consistente, el estímulo que señalizaba el IF vigente durante la fase de prueba fue el mismo que se utilizó durante la adquisición y la extinción; mientras que para el grupo inconsistente, el estímulo correlacionado a cada IF fue diferente al utilizado durante la fase de adquisición y extinción. Durante la fase de prueba, los autores reportaron en ambos grupos un incremento en la tasa de respuesta en comparación con la fase de extinción. Sin embargo, la distribución temporal del responder solo se recuperó en los sujetos del grupo consistente.

Haber observado una recuperación tanto de la tasa de respuesta como de la distribución temporal, deja ver que las claves o señales contextuales no controlan únicamente la tasa, sino que también son moduladoras de la distribución temporal del responder. La importancia de este punto recae en cómo permite reconocer que la ejecución está compuesta tanto por la tasa como por la distribución, siendo consistente con lo señalado por Ferster y Skinner (1957) así como lo reportado por Gilbert (1958), Guilhardi y Church, (2006), Gentry et al. (1983) quienes reconocen que además de la tasa, también hay otras propiedades de la respuesta que pueden ser sensibles a las manipulaciones realizadas, en este caso la distribución temporal del responder en preparaciones para el estudio 
de la renovación contextual. Es decir, los resultados presentan una forma alternativa para evaluar la magnitud del efecto de renovación contextual en diversas manipulaciones (e.g., número de sesiones, cualidad del reforzador, etc.). Además de que estos resultados plantean la pregunta ¿Qué distribución temporal del responder (e.g., una distribución positivamente acelerada producida por un IF o una distribución lineal producida por un IV) genera mayor o menor renovación contextual? Estas y otras preguntas se desarrollan en el laboratorio donde se han realizado las pruebas de este estudio. Responder estas interrogantes permitirá ahondar en las explicaciones que han sido elaboradas al considerar también la dimensión temporal del comportamiento, debido a como ya lo han señalado otros investigadores (Gentry et al., 1983) se debe tener cuidado con las interpretaciones basadas simplemente en la tasa de respuestas, debido a la limitación en su valor.

Los presentes resultados, son consistentes con las explicaciones conceptuales que se han ofrecido entorno a la renovación contextual. Por ejemplo el modelo de recuperación de información de Bouton $(1993 ; 1994)$ plantea que los organismos durante la fase de adquisición forman un aprendizaje, el cual no desaparece durante la fase de extinción, sino que, durante la fase de extinción se establece un nuevo tipo de aprendizaje. El aprendizaje durante la fase de adquisición y el aprendizaje durante la fase de extinción resultan en una situación de ambigüedad que es eliminada por las claves o señales contextuales. De esta manera, el modelo supone que para observar renovación durante la fase de prueba se debe realizar en un contexto diferente al empleado en la fase de extinción, como se muestran en los resultados del presente estudio en el Grupo ABA.

En su conjunto, los resultados del experimento permitieron identificar las condiciones necesarias para observar la renovación contextual de la ejecución empleando un programa de IF, posibilitando hacer réplicas y comparaciones al observar la generalidad de un fenómeno (Sidman, 1960), en este caso la renovación contextual tanto de la tasa de respuesta como la de la distribución temporal del responder.

\section{Agradecimientos}

El presente trabajo forma parte de los estudios realizados por el primer autor para obtener el grado de Maestro en Ciencia del Comportamiento bajo la dirección del segundo.

\section{Referencias}

Bernal-Gamboa, R., Carrasco-López, M., \& Nieto, J. (2014). Contrasting ABA, AAB and $\mathrm{ABC}$ renewal in a free operant procedure. Spanish Journal of Psychology, 17, 01-06. http://doi.org/10.1017/sjp.2014.68

Bouton, M. (1993). Context, time and memory retrieval in the interference paradigms of pavlovian learning. Psychological Bulletin, 114(1), 80-99. https://doi.org/10.1037/003 3-2909.114.1.80

Bouton, M. (1994). Conditioning, remembering and forgetting. Journal of Experimental Psychology: Animal Behavior Processes, 20(3), 219-231. https://doi.org/10.1037/00 97-7403.20.3.219

Bouton, M. \& Bolles, R. (1979). Contextual control of the extinction of conditioned fear. Learning and Motivation 10(4), 445-466. https://doi.org/10.1016/0023-969 0(79)90057-2

Bouton, M., Todd, T., Vurbic, D., \& Winterbauer, N. (2011). Renewal after the extinction of free operant behavior. Learning $\mathcal{E}$ Behavior, 39(1), 57-67. https://doi.org/10.3758/s134 20-011-0018-6

Cabrer, F. (1987). Efectos de la duración de un reloj externo sobre un programa de intervalo fijo. Revista Mexicana de Análisis de la Conducta, 13(1-2), 153-172. Recuperado de http://revistas.unam.mx/index.php/rma c/article/download/26910/25086

Cançado, C. \& Lattal, K. (2011). Resurgence of temporal patterns of responding. Journal of the Experimental Analysis of Behavior, 95(3), 
271-287. https://doi.org/10.1901/jeab.2011 $.95-271$

Cuevas, K., Learmonth, A., \& Roveer-Collier, C. (2015). A dissociation between recognition and reactivation: The renewal effect at 3 months of age. Developmental Psychobiology, 58(2), 159-175. https://doi.org/10.1002/de v. 21357

Delamater, A. (2016, abril). On the relative permanence of memories processes involved in the acquisition and extinction of pavlovian learning. Conferencia: Andrew Delamater (CUNY, Brooklyn College). Guadalajara: Centro de Estudios e Investigación en Comportamiento. Recuperado de http://www.ceic.cucba.udg. $\mathrm{mx} /$ Actividades/generales? $\mathrm{id}=305$

Dews, P. (1969). Studies on responding under fixed-interval schedules of reinforcement: the effects on the pattern of responding of changes in requirements at reinforcement. Journal of the Experimental Analysis of Behavior, 12(2), 191-199. https://10.1901/je ab.1969.12-191

Eddy, M., Todd, T., Bouton, M. \& Green, J. (2016). Medial prefrontal cortex involvement in the expression of extinction and ABA renewal of instrumental behavior for a food reinforce. Neurobiology of Learning and Memory, 128, 33-39. https://doi.org/10. 1016/j.nlm.2015.12.003

Ferster, C.B. \& Skinner, B.F. (1957). Schedules of Reinforcement. New York: Appleton Century Crofts.

Gentry, G. D., Weiss, B., \& Laties, V. G. (1983). The microanalysis of fixed-interval responding. Journal of the Experimental Analysis of Behavior, 39(2), 327-343. Recuperado de http://psycnet.apa.org/recor d/1983-29551-001

Gilbert, T. F. (1958). Fundamental dimensional properties of the operant. Psychological Review, 65(5), 272-282. https://doi.org/10. $1037 / \mathrm{h} 0044071$

Guilhardi, P., \& Church, R. M. (2004). Measures of temporal discrimination in fixed-interval performance: a case study in archiving data. Behavior Research Methods, Instruments, $\mathcal{E}$
Computers, 36(4), 661-669. https://doi.org/ 10.3758/BF03206548

Guilhardi, P., \& Church, R. (2006). The pattern of responding after extensive extinction. Learning E Behavior, 34(3), 269-284. https ://doi.org/10.3758/BF03192883

López, F., \& Menez, M. (2005). Effects of reinforcement history on response rate and response pattern in periodic reinforcement. Journal of the Experimental Analysis of Behavior, 83(3), 221-241. https://doi.org/10 $.1901 /$ jeab.2005.49-04

Kearns, D. \& Weiss, S. (2007). Contextual renewal of cocaine seeking in rats and its attenuation by the conditioned effects of an alternative reinforce. Drug and Alcohol Dependence, 90(2-3), 193-202. https://doi.o $\mathrm{rg} / 10.1016 / \mathrm{j}$.drugalcdep.2007.03.006

Kelley, M., Linddon, C., Ribeiro, A., Greif, A. \& Podlesnisk, C. (2015). Basic and translational evaluation of renewal of operant responding. Journal of Applied Behavior Analysis, 48(2), 390-401. https://d oi.org/10.1002/jaba.209

Nakajima, S., Tanaka, S., Urushihara, K., \& Imada, H. (2000). Renewal of extinguished lever-Press responses upon return to the training context. Learning and Motivation, 31 (4), 416-431. https://doi.org/10.1006/lm ot.2000.1064

Podlesnik, C. \& Miranda-Dukoski, L. (2015). Stimulus generalization and operant context renewal. Behavioural Processes, 119, 93-98. http://doi.org/10.1016/j.beproc.201 5.07.015

Podlesnik, C., Kelley, M., Jimenez-Gomez, C. $\&$ Bouton, M. (2017). Renewed behavior produced by context change and its implications for treatment maintenance: A review. Journal of Applied Behavior Analysis, 50(3), 675-697. http://doi.org/10.1002/ jaba. 400

Sidman, M. (1960). Tactics of scientific research: Evaluating experimental data in psychology. New York: Basic Books.

Skinner, B. F. (1938). The Behavior of Organisms. Oxford: Appleton Century Crofts. 
Sweeney, M. \& Shahan, T. (2015). Renewal, resurgence, and alternative reinforcement context. Behavioural Processes, 116, 43-49. h ttp://doi.org/10.1016/j.beproc.2015.04.015

Todd, T., Winterbauer, N. \& Bouton, M. (2012). Effects of the amount of acquisition and contextual generalization on the renewal of instrumental behavior after extinction. Learning $\mathbb{E}$ Behavior, 40(2), 145-157. http:/ /doi.org/10.3758/s13420-011-0051-5

Trask, S. \& Bouton, M. (2016). Discriminative properties of the reinforcer can be used to attenuate the renewal of extinguished operant behavior. Learning $\mathfrak{G}$ Behavior, 44(2), 151-161. http://doi.org/10.3758/s13 420-015-0195-9

Vurbie, D., Gold, B. \& Bouton, M. (2011). Effects of D-Cycloserine on the extinction of appetitive operant learning. Behavioral Neuroscience, 125(4), 551-559. http://doi.o $\mathrm{rg} / 10.1037 / \mathrm{a} 0024403$

\section{Notas}

* Artículo de investigación. 